\title{
A framework for smartphone-enabled, patient-generated health data analysis
}

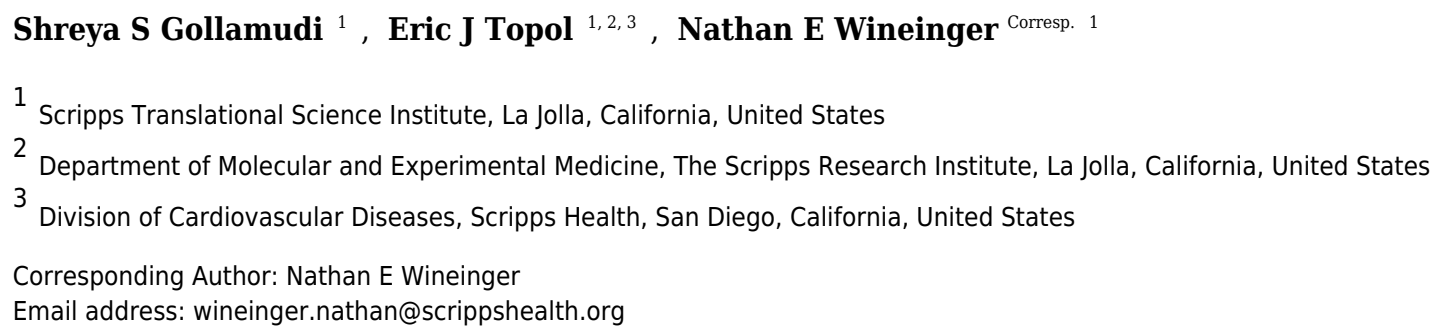

Background: Digital medicine and smartphone-enabled health technologies provide a novel source of human health and human biology data. However, in part due to its intricacies, few methods have been established to analyze and interpret data in this domain. We previously conducted a six-month interventional trial examining the efficacy of a comprehensive smartphone-based health monitoring program for individuals with chronic disease. This included 38 individuals with hypertension who recorded 6,290 blood pressure readings over the trial. Methods: In the present study we provide a hypothesis testing framework for unstructured time series data, typical of patient-generated mobile device data. We used a mixed model approach for unequally spaced repeated measures using autoregressive and generalized autoregressive models, and applied this to the blood pressure data generated in this trial. Results: We were able to detect, roughly, a $2 \mathrm{mmHg}$ decrease in both systolic and diastolic blood pressure over the course of the trial despite considerable intra- and inter-individual variation. Furthermore, by supplementing this finding by using a sequential analysis approach, we observed this result over three months prior to the official study end - highlighting the effectiveness of leveraging the digital nature of this data source to form timely conclusions. Conclusions: Health data generated through the use of smartphones and other mobile devices allow individuals the opportunity to make informed health decisions, and provide researchers the opportunity to address innovative health and biology questions. The hypothesis testing framework we present can be applied in future studies utilizing digital medicine technology or implemented in the technology itself to support the quantified self. The study was registered at clinicaltrials.gov (NCT01975428). 
1

61 Scripps Translational Science Institute, La Jolla, California, USA

72 Department of Molecular and Experimental Medicine, The Scripps Research Institute, La Jolla,

8 California, USA

$9{ }^{3}$ Division of Cardiovascular Diseases, Scripps Health, San Diego, California, USA

10

11 Nathan E. Wineinger, Ph.D.

12 The Scripps Translational Science Institute

133344 North Torrey Pines Court, Suite 300

$14 \quad$ La Jolla, CA 92037

$15 \quad$ (858) 554-5728

16 wineinger.nathan@scrippshealth.org 
18 ABSTRACT

19 Background: Digital medicine and smartphone-enabled health technologies provide a novel

20 source of human health and human biology data. However, in part due to its intricacies, few

21 methods have been established to analyze and interpret data in this domain. We previously

22 conducted a six-month interventional trial examining the efficacy of a comprehensive

23 smartphone-based health monitoring program for individuals with chronic disease. This included

2438 individuals with hypertension who recorded 6,290 blood pressure readings over the trial.

25 Methods: In the present study we provide a hypothesis testing framework for unstructured time 26 series data, typical of patient-generated mobile device data. We used a mixed model approach

27 for unequally spaced repeated measures using autoregressive and generalized autoregressive 28 models, and applied this to the blood pressure data generated in this trial.

29 Results: We were able to detect, roughly, a $2 \mathrm{mmHg}$ decrease in both systolic and diastolic blood pressure over the course of the trial despite considerable intra- and inter-individual

31 variation. Furthermore, by supplementing this finding by using a sequential analysis approach,

32 we observed this result over three months prior to the official study end - highlighting the

33 effectiveness of leveraging the digital nature of this data source to form timely conclusions.

34 Conclusions: Health data generated through the use of smartphones and other mobile devices 35 allow individuals the opportunity to make informed health decisions, and provide researchers 36 the opportunity to address innovative health and biology questions. The hypothesis testing

37 framework we present can be applied in future studies utilizing digital medicine technology or 38 implemented in the technology itself to support the quantified self. The study was registered at 39 clinicaltrials.gov (NCT01975428). 


\section{INTRODUCTION}

43 Empowered patients and health care consumers (Topol, 2015) have aligned with health data 44 tracking technologies to create the quantified self movement (Swan, 2013). Quantified self 45 involves the use of tracking one's own health-related data to understand trends and potentially 46 alter behavior in order to achieve a health goal. The size and scope of health tracking has and 47 continues to expand with the advent of digital medicine and mobile health (mHealth) 48 technologies enabled by smartphones and connected device infrastructures (Steinhubl, Muse \& 49 Topol, 2015). For example, in certain individuals traditional daily weight monitoring has been 50 supplemented by apps that track food intake and devices that monitor physical activity in order

51 to achieve this health goal - a part of the $58 \%$ of mobile phone users that have downloaded a 52 health-related mobile app (Krebs \& Duncan, 2015). Researchers have shown that in some cases interventions using this technology alone can improve health outcomes, though this result is far from universal (see (Free et al., 2013) for review) with the disparity likely due to numerous factors including poor adherence and fatigue (Shaw et al., 2016), and failure of the intervention to change behavior (Patel, Asch \& Volpp, 2015).

While new, better, more user-friendly technologies will continue to be developed, vying for the appreciable forecasted growth of the industry (Statista, 2016), we and others believe the future of the field is not simply in the devices and software themselves, but in the data they generate

61 (Gibbs, 2015). Such data can help guide individual health decisions - the crux of the quantified

62 self movement - but can also be used to address novel human health and biology questions

63 (Steinhubl et al., 2015). Yet there exists a sizable gap between the data that is generated and 64 the methods available to analyze and interpreted such data (Fawcett, 2015). Indeed, most such 65 devices and apps simply display the data, leaving any inference up to the user and anyone the 66 user wishes to share the data with (e.g., their physician). However, even the most data 67 experienced users and health care providers may find identifying subtle trends in such complex 
68 data a daunting task. These challenges also extend to researchers who may wish to examine

69 data captured from these technologies as, in addition to inherent technical obstacles such as

70 data collection and data security, few analytic methods are established and general software

71 packages are not readily available.

72

73 These technologies present the opportunity to examine health data in nontraditional ways.

74 Rather than large intermittent gaps in health measures between doctor or study visits, data can

75 be collected in relatively high resolution. Such high resolution data allows users and researchers

76 to detect unique trends and relationships that were never before possible. For example, a

77 diabetic patient using a continuous glucose monitor can now assess their minute-to-minute

78 health rather than rely on single, low resolution measures such as hemoglobin A1c levels, which

79 does not accurately assess health variability (Virtue et al., 2004). Furthermore, access to such

80 high-resolution human health data in nontraditional settings (e.g., normal, at home environment)

81 allows us to evaluate "real world" health and not be relegated to artificial worlds created in

82 clinical trials that suffer from poor clinical practice adoption (Goss, Elmore \& Lessler, 2003). Yet

83 in order to form scientific conclusions in this new frontier, novel methods and adaptations of

84 existing approaches must be developed to account for the intricacies of patient-generated data.

86 While numerous digital medicine biosensors, devices, and applications have been

87 manufactured to measure various aspects of human physiology and exposome, perhaps no

88 metric epitomizes both the contemporary challenge and opportunity of this field more than blood

89 pressure. Heart disease is the leading cause of death in the United States, with hypertension

90 being the leading contributor of disease (National Center for Health Statistics, 2012). With

91 proper management, hypertension can be controlled and the health consequences of

92 uncontrolled hypertension can largely be avoided. Nevertheless, in the United States only $48 \%$

93 of individuals with hypertension have their condition under control (Farley et al., 2010). It is 
94 unsurprising that hypertension has been a target for patient-centric, mHealth disease

95 management (Logan, 2013). Yet the technology to continuously monitor blood pressure in the

96 outpatient setting is still developing as manufacturers have not entirely solved the technical

97 aspects of truly passive monitoring. Thus, the current state of the field largely includes mobile

98 blood pressure cuffs in which readings are initiated by the user. As users may take a reading at

99 any time, evaluating temporal trends in this data requires added consideration.

101 In this study we present a hypothesis testing framework in which we examined blood pressure 102 readings taken at variable, uncontrolled time points in individuals enrolled in a smartphone-

103 based health monitoring intervention trial - though the approach we present can be adapted to

104 other similarly structured data. In total, 38 study participants recorded and provided us with 105 blood pressure data on 6,290 occasions. We find that by leveraging all data across individuals 106 we were able to detect an approximately $2 \mathrm{mmHg}$ decrease in blood pressure over a 6 month 107 trial, despite considerable intra- and inter-individual variation. We then discuss how this and 108 other techniques can be implemented in data analyses of the quantified self and in future study 109 designs.

\section{METHODS}

\section{Study participants}

113 The present investigation is a sub-analysis of a study conducted by the Scripps Translational

114 Science Institute named the Wired for Health (WFH) study (Bloss et al., 2016). In brief, the WFH 115 study was a 6 month, randomized-controlled trial investigating the practice of a smartphone116 based health monitoring program in individuals with chronic disease, and was accompanied by 117 an online and mobile tracking infrastructure. Eligible participants were over the age of 18 who 118 were insured by Scripps Health and had submitted at least one health insurance claim for 119 hypertension, diabetes, or cardiac arrhythmia in 2012. Participants were equally randomized to 
120 the control or monitoring arms (details below). In total, 160 participants enrolled in the WFH

121 study, with the majority (51.3\%) being in the top quartile of health insurance claims in 2012.

122 Only individuals with hypertension $(n=135)$ were considered in the present study. Among those,

123112 completed the study, including 53 in the control and 59 in the monitoring group. After

124 screening device readings data for technical limitations $(n=19)$ and study noncompliance $(n=2)$,

12538 hypertensive individuals from the monitor group had complete readings data. This study

126 focuses on these 38 individuals. This study was approved by the Scripps Institutional Review

127 Board (IRB-12-6019), and all study participants provided informed consent.

128

129 Monitoring intervention arm

130 Hypertensive study participants in the monitoring group were provided with comprehensive

131 mobile blood pressure monitoring system: a Withings Blood Pressure Monitor, an iPhone 4 or

132 4s with linked applications, iPhone applications, and an online and mobile HealthyCircles

133 account. HealthyCircles is a Qualcomm Life health care coordination and management platform

134 with an integrated suite of management and consumer portals that can deliver chronic disease

135 education and connect users to their families, caregivers, and health care professionals.

136 Individuals were instructed to measure their blood pressure using this system twice a day, three

137 days a week, with the first measurement in the morning. If participant measurements dropped

138 below a desired level of compliance (less than three measurements a week for two consecutive

139 weeks) the participant was sent an email through their HealthyCircles account reiterating the

140 measurement schedule. Participants were also encouraged to take extra measurements if

141 deemed appropriate. Device readings data was collected using Qualcomm Life's cloud-to-cloud

142 data integration capability.

143

\section{Variables of interest}


145 The primary outcome of the present study was device-collected blood pressure measurements

146 in the 38 individuals with complete readings data in the monitoring group. The primary

147 independent variable is time since the beginning of enrollment in the study. The hour during the

148 day the measurement was taken was considered as a covariate.

150 Statistical Analyses

151 Device readings data was analyzed using two approaches: 1) a multiple N-of-1 approach in

152 which the data from each study participant was analyzed individually; and 2) a mixed model

153 approach combining all individuals for analysis. Specific details on each model are available in

154 Supplemental Appendix 1.

155

156 In the multiple N-of-1 approach, blood pressure measures were regressed on time enrolled in

157 the study using linear regression, accounting for the time of day at which the measurements

158 were taken. Alternative covariance structures were modeled, but results were consistent with

159 those obtained from simple linear regression. In all cases, the effect of blood pressure over time

160 (i.e. slope) was recorded. Slope averages, inverse variance weighted averages, and

161 bootstrapped confidence intervals were calculated.

162

163 Alternatively, repeated measures mixed models were constructed to assess blood pressure 164 over time across all individuals. The general structure of the model is:

$$
\mathbf{Y}=\mathbf{X} \boldsymbol{\beta}+\mathbf{Z u}+\mathbf{e}
$$

166

where $\mathbf{Y}$ is the vector of blood pressure measures, $\mathbf{X}$ is a matrix of independent variables (i.e.

167 intercept, time, and covariates) with fixed effects $\boldsymbol{\beta}, \mathbf{Z}$ is a matrix indicating the structure of the

168 between subject random effects $\mathbf{u}$ with covariance matrix $\mathbf{G}$, and $\mathbf{e}$ is the random error with

169 covariance matrix $\mathbf{R}$. It follows that $\operatorname{Var}(\mathbf{Y} \mid \mathbf{u})=\mathbf{R}$ and $\operatorname{Var}(\mathbf{Y})=\mathbf{V}=\mathbf{Z G Z} \mathbf{Z}^{\mathrm{T}}+\mathbf{R}$. 
171 In the present study, $\mathbf{R}$ represents the potential time-dependency of measures within subjects.

172 It follows that $\mathbf{R}$ and subsequently $\mathbf{V}$ are block diagonal. Below we refer to the block diagonal

173 elements of $\mathbf{V}$ as $\boldsymbol{\Sigma}$, and the elements of $\mathbf{R}$ as $\boldsymbol{\Sigma}_{\mathrm{R}}$. The primary hypothesis tested was

$174 \mathrm{H}_{0}: \beta_{\text {time }}=0$. That it, was there a linear change in blood pressure measures in the study

175 population after accounting for individual variation and potential time-dependency between

176 measures? Time of day (in hours) was modeled as a fixed effect covariate with 24 levels. We do

177 note that that random effects component of this model indirectly accounts for static individual-

178 specific covariates (e.g., baseline BMI). Thus, no other covariates were modeled.

180 Three distinct covariance structures were modeled that were appropriate for the source of the

181 data and minimally complex on account of the large dimensions of $\mathbf{V}: 1$ ) compound symmetric

182 structure $\boldsymbol{\Sigma}$ (i.e. random effects only); 2) a first-order autoregressive structure with random

183 effects; and 3) a spatial power law/generalized autoregressive structure with random effects.

184 Model fit was assessed using AIC and BIC.

186 Finally, a sequential analysis approach was implemented using the mixed model approach

187 described above. Study device readings data was collected over a roughly ten and one-half

188 month period from the middle of August 2013 to July 2014. Data was partitioned into eleven

189 cumulative monthly periods. For example, one data partition included all device readings data

190 from the first month of the study, another included device readings data from the first two

191 months of the study, and so on. A mixed model with spatial power law covariance structure with

192 random effects was applied to each of the data partitions. Additionally, a sequential analysis

193 approach was implemented in an N-of-1 framework where readings from each individual alone

194 were partitioned into monthly blocks similar to that described above. Among individuals who did 
195 demonstrate a significant increase or decrease in blood pressure at six months, the goal was

196 determine if changes in blood pressure could be observed prior to the conclusion of the

197 individual's study participation (e.g., if an individuals demonstrated a decrease in blood pressure

198 over 6 months, would we have observed that result after 5 months). Likewise, a spatial power

199 law covariance structure was assumed.

200

201

\section{RESULTS}

202 Demographic information on study participants is presented in Supplemental Table 1. Among

203 the 59 original study participants assigned to the monitoring intervention arm that completed the

204 study, 38 had complete device readings data. This cohort was predominantly Caucasian (87\%)

205 and largely female (74\%) with an average age of 57 . A number of participants did not own a

206 smartphone prior to enrollment in the study (21\%) with half owning an iPhone. Responses to

207 health-related survey questions are presented in Supplemental Table 2. We saw a general

208 increase in overall health by the end of the study. There was notable decrease in smoking

209 frequency and increase in exercise frequency. However, we note that these health

210 improvements were also observed in the control arm of the original study (data not presented),

211 suggesting that the monitoring intervention itself had no discernible impact on these traits.

212

213 The implied goal of individuals participating in the study is better management of their condition.

214 In this regard, we considered observed blood pressure device readings collected over the

215 course of the study as outcomes of interest. Single blood pressure readings taken at, for

216 example, the enrollment and end of study visit can provide some level of inference on blood

217 pressure changes. However, this approach ignores all data that could be generated between

218 these time points and is vulnerable to biases and natural variation. Rather, we feel approaches

219 which leverage the entirety of the data are preferential. By utilizing Qualcomm's cloud-to-cloud

220 data integration capability, we were able to capture the measure and time recorded of each 
221 blood pressure reading on 38 individuals in the monitoring group. While our inference is based

222 on these 38 individuals and is limited, we present our mathematical framework and modeling

223 below in attempt to answer the seemingly simple question: was blood pressure changing over

224 the course of the study?

225

226 In total, we collected 6,290 systolic and 6,265 diastolic blood pressure readings from these

227 individuals (Supplemental Data 1, Supplemental Data 2). Device readings were recorded 228 roughly uniform over the course of the study (Supplemental Figure 1). The number of readings

229 taken varied between individuals, with an average of 165 readings per person ( $\mathrm{sd}=70, \mathrm{~min}=61$,

$230 \max =416)$. The time of day that readings were taken was also variable, with a large proportion

231 of measurements taken in the early morning and afternoon. Few readings were taken in the late

232 morning and at night, though this was not necessarily surprising given we asked participants to

233 use the device in the morning, presumably before day time activities (Supplemental Figure 2). 234

235 Multiple N-of-1 approach

236 We first assessed the effect of time on blood pressure on each study participant individually

237 (Figure 1, Supplemental Figure 3). Among the 38 participants, 18 had nominally statistically 238 significant $(p<0.05)$ changes in systolic blood pressure and 21 had significant changes in 239 diastolic blood pressure. However, the number of participants with a significant decrease in 240 systolic blood pressure $(n=9)$ was equal to the number with an increase $(n=9)$, and was similarly 241 true for diastolic blood pressure (decrease: $n=12$, increase: $n=7 ; p=0.36$ ). This result also held 242 when we examined the estimated effects from all participants, regardless of $p$-value. There were 24320 individuals with a decrease (i.e. negative slope) in systolic blood pressure against 18 with an 244 increase $(p=0.87)$, and 21 with a decrease and 17 with an increase in diastolic blood pressure $245(p=0.62)$. In efforts to summarize these results across individuals, we calculated the mean slope 246 and mean slope weighted by the square root of the number of readings each participant 
247 recorded. We found no evidence towards an overall decrease in systolic or diastolic blood 248 pressure. The weighted mean change in systolic blood pressure was $-1.7 \mathrm{mmHg}(95 \% \mathrm{Cl}:-4.7$, 249 1.4) and diastolic blood pressure was $-1.9(95 \% \mathrm{Cl}:-4.0,0.1)$. Results from the unweighted 250 calculations were similar.

251

252 Mixed model approach

253 We then pooled device readings data from all study participants, and assessed the effect of time 254 on blood pressure using the mixed model framework described previously with three possible 255 covariance structures of $\mathbf{R}$, where $\boldsymbol{\Sigma}_{\mathrm{R}}$ was 1) compound symmetric; 2) first-order 256 autoregressive; or 3) generalized autoregressive for unequally spaced data (i.e. spatial power 257 law).

258

259 We encountered one issue when applying the spatial power law structure mixed model. As 260 opposed to the first-order autoregressive model in which

$$
\Sigma_{\mathrm{R}}=\sigma^{2}\left(\begin{array}{cccc}
1 & \rho & \rho^{2} & \\
\rho & 1 & \rho & \mathrm{L} \\
\rho^{2} & \rho & 1 & \\
& \mathrm{M} & & \mathrm{O}
\end{array}\right)
$$

262 where $\rho$ is the correlation between two successive measures on the same subject, the spatial 263 power law structure is a generalized form of the first-order autoregressive model in which

$$
\Sigma_{\mathrm{R}}=\sigma^{2}\left(\begin{array}{cccc}
1 & \rho^{t_{2}-t_{1}} & \rho^{t_{3}-t_{1}} & \\
\rho^{t_{2}-t_{1}} & 1 & \rho^{t_{3}-t_{2}} & \mathrm{~L} \\
\rho^{t_{3}-t_{1}} & \rho^{t_{3}-t_{2}} & 1 & \\
& \mathrm{M} & & \mathrm{O}
\end{array}\right)
$$

265 where $t_{k}$ is the time of the $k^{\text {th }}$ measurement, and the difference $t_{i}-t_{j}$ is the lag between the $266 i^{\text {th }}$ and $j^{\text {th }}$ measure. When measurements are equally spaced, the spatial power law models 
267 simplifies to a first-order autoregressive model as the lag between measures is constant.

268 Though we instructed study participants to measure their blood pressure at certain intervals, the

269 time individuals chose to take these readings, and thus the lag between measures, varied

270 considerably. In this context, we expected the spatial power law structure to be appropriate for

271 the data collected. However, while the majority of consecutive measures had lags of six hours

272 or more (Figure 2), we discovered a small number of consecutive measures with short lag that

273 led to issue we alluded to above. To demonstrate this issue between consecutive

274 measurements with short lag, consider the following example: three measurements are

275 recorded at times labeled $t_{1}, t_{2}$, and $t_{3}$. In this case,

276

$$
\boldsymbol{\Sigma}_{\mathrm{R}}=\sigma^{2}\left(\begin{array}{ccc}
1 & \rho^{t_{2}-t_{1}} & \rho^{t_{3}-t_{1}} \\
\rho^{t_{2}-t_{1}} & 1 & \rho^{t_{3}-t_{2}} \\
\rho^{t_{3}-t_{1}} & \rho^{t_{3}-t_{2}} & 1
\end{array}\right) .
$$

277 However, when two measurements are taken relatively close together, say at $t_{1}$ and $t_{2}$ 278 compared to $t_{3}$, then $t_{2}-t_{1}$ is relatively close to zero and $t_{3}-t_{1} \approx t_{3}-t_{2}$. The result on $\Sigma_{\mathrm{R}}$ is:

$$
\boldsymbol{\Sigma}_{\mathrm{R}} \approx \sigma^{2}\left(\begin{array}{ccc}
1 & 1 & \rho^{t_{3}-t_{1}} \\
1 & 1 & \rho^{t_{3}-t_{1}} \\
\rho^{t_{3}-t_{1}} & \rho^{t_{3}-t_{1}} & 1
\end{array}\right) .
$$

As can be seen, the first and second columns and rows are roughly equivalent, leading to singularity in this matrix, non-convergence, and inestimable effects. It should be noted that this issue does not manifest in the compound symmetric and first-order autoregressive models.

284 In order to solve this issue, we merged data readings taken within short periods of each other by taking the average over that time. We found that merging readings within a one hour period of 286 each other eliminated the singularity of $\Sigma_{R}$ while minimizing the number of readings 287 manipulated. We still ran into singularity issues when using shorter time intervals (e.g., 15 
288 minute and 30 minute minimums). In total, we merged 826 systolic and 801 diastolic blood

289 pressure readings within an hour of each other, with most merging (61\%) being two readings

290 recorded within an hour. The subsequent dataset resulted in 5,464 systolic and diastolic

291 readings.

292

293 Mixed model results from the one-hour merged dataset are presented in Table 1. For each

294 model assessed, there was an approximately $2 \mathrm{mmHg}$ decrease in systolic and diastolic blood 295 pressure across the sample over the study period ( $p<0.001$ in all cases). While both the first-

296 order autoregressive and spatial power law models with random effects had better model fit

297 than the random effects model alone, the first-order autoregressive model had slightly better

298 model fit (the estimate of $\rho$ was near 0.2 regardless of the approach). It should be noted that

299 when we tested various merging strategies to eliminate the singularity in the spatial power law

$300 \Sigma$ matrix (e.g., averaging blood pressure across the entire day) sometimes the spatial power

301 law model had the better fit. This suggests that while accounting for time dependencies was

302 important, the precise structure appeared to be less so. This was true even though the spatial

303 power law model appeared to be more appropriate, given the readings were generally unequally

304 spaced.

305

306 Sequential analysis approach

307 We next implemented a sequential analysis approach in which we modeled a spatial power law

308 structure with random effects on sequential subsets of the one-hour merged datasets. The goal

309 of this approach was to determine if and when we could have arrived at our primary conclusion

310 (i.e. systolic and diastolic blood pressure decrease $2 \mathrm{mmHg}$ over the study) prior to the end of

311 the study. While the device readings data across all study participants was collected over ten

312 and a half months, after the first seven months of the study we would have arrived at a similar 
313 conclusion (Figure 3, Supplemental Figure 4). Though all 38 participants had completed the

314 study or were enrolled by that time, and 4,686 blood pressure measures ( $86 \%)$ had taken place

315 by then, the prospect of arriving at a conclusion prior to the designed end of the study has some

316 benefit - particularly so when data is collected using digital medicine devices as we discuss

317 below. Furthermore, when applying a sequential analysis approach in an N-of-1 framework, we

318 were able to determine that 13 of the 15 individuals with nominally significant systolic blood

319 pressure changes, and 14 of the 18 individuals with diastolic blood pressure changes over 6

320 months demonstrated this trend $(p<0.05)$ by the fifth month of their study enrollment.

321

\section{DISCUSSION}

323 As digital, smartphone-enabled, and other patient-centric medical and health technologies have

324 the potential to improve individual health and the overall health care system, the quest to 325 develop the "best" technologies will remain ongoing. However, we and others feel the future of 326 this field is not simply in the devices, sensors, software, and wearables per se (Gibbs, 2015),

327 but in what the data generated from these tools can tell us about human health and biology.

328 Above we present a framework for hypothesis testing on unequally spaced time series data - a

329 common feature of data generated from these technologies. Applied to a subset of hypertensive

330 individuals enrolled in an interventional trial, we discovered that individuals participating had, on

331 the whole, a roughly $2 \mathrm{mmHg}$ decrease of systolic and diastolic blood pressure over a 6 month

332 period. Using the methods presented we were able to observe this distinction despite

333 considerable intra and inter-individual variation in blood pressure measures, and without a

334 rigorously structured readings schedule.

336 The context of this discovery should, however, be considered. As stated above, we detected a

337 statistical increase in systolic $(n=9)$ and diastolic blood pressure $(n=7)$ in some study 338 participants, while device readings were (unsurprisingly) quite variable even among study 
339 participants that clearly showed a trend toward lowering blood pressure. We have recently

340 demonstrated some explanation for this heterogeneity in study outcomes (Kim, Wineinger \&

341 Steinhubl, 2016). But this observation demonstrates the advantage, yet caution, needed when

342 performing analyses and interrupting results that leverage large amounts of high-dimensional

343 patient-generated data. On one hand, this presents an opportunity to identify subtle trends in

344 health data that might otherwise be difficult to observe - such as single measurements

345 intermittently collected during a physician visit. On the other hand, large amounts of data can

346 make it possible to detect any trend even if this trend does not substantially contribute to our

347 knowledge of human health and biology. For these reasons we find it more informative to reflect

348 on the magnitude of the effect and less on the magnitude of the $p$-value. Complementary

349 approaches which specify the 'null' model as a minimally meaningful effect (i.e., non-zero

350 contrast matrix) may be more appropriate in such cases.

351 Importantly, while we focused on time enrolled in the study as the independent variable of

352 interest, the framework we present can also be adapted to examine other temporal relationships

353 in similarly generated data. For example, this framework can be used to compare data captured

354 between discrete conditions such as intervention versus control, or to differentiate based on

355 other quantitative measures. In these cases, the $\mathbf{X}$ matrix presented above can be remodeled

356 to reflect the desired design matrix. Additionally, while our mixed model approach modeled data

357 across all 38 study participants collectively, this framework can be also adapted to examine

358 temporal trends on a single individual, such as that of an N-of-1 crossover design or how we

359 examined individual trends in the $\mathrm{N}$-of-1 sequential analysis approach above. In this case, the

$360 \mathbf{Z}$ matrix can be omitted on account of no between subject effects, while $\mathbf{R}$ would remain.

362 One of the more intriguing aspects of this technology as a tool to enhance individual health is

363 that data is collected, stored, and presented digitally without the need for direct interaction

364 between the user and (as traditional) health professional. Likewise, we feel the processes of 
365 data inference can also be built into the technology to bypass the need for data interpretation by

366 a professional data analyst. Certainly many technologies have such tools. Yet as new methods

367 and extension of existing approaches, such as the framework we presented, are developed,

368 these will need to be implemented into the technology in order to provide users with the best

369 opportunity to make informed health decisions based on this data. The most immediate way this

370 can be accomplished is by coding these methods directly on the device or software, or

371 accessible to a cloud server where computations can be performed. However, other options

372 include crowd-sourced initiatives such as an app store, where the public can design specialized

373 software which provides automated analyses and interpretation of data back to the user.

374

375 Alternatively, the digital nature of data obtained from this technology opens up a number of 376 interesting possibilities for researchers. Again, because data can be continuously collected 377 without the need for personnel (e.g., study coordinators) to interact with users/study 378 participants, approaches which benefit from data analyses over the course of the study may 379 prove beneficial. We attempted to show this in the sequential analysis approaches above in 380 which we were able to arrive at the primary study result over three months prior to the end of the 381 study. Methods like this can be implemented directly into the study design, such as those in 382 adaptive clinical trials. Moreover, because data on individuals can be recorded, collected, 383 analyzed, and interpreted in real time, concepts such as early stoppage due to success or futility 384 can apply not only to the study itself, but study participants as well - thereby minimizing risks, 385 reducing costs, and forming conclusions earlier.

387 Data collected from digital medicine and smartphone-enabled health technologies offers 388 tremendous potential to learn more about human health and biology. We applaud 389 manufacturers for striving towards more comprehensive monitoring technologies, and when 
390 applicable encourage researchers to use this data source to help address research questions of

391 interest.

392

393 ACKNOWLEDGEMENTS

394 Steven R. Steinhubl, M.D. provided study suggestions; Qualcomm Life made device readings

395 data available.

396 
397

398

399

400

401

402

403

404

405

406

407

408

409

410

411

412

413

414

415

416

417

418

419

420

421

422

423

424

425

426

427

428

429

430

431

432

\section{REFERENCES}

Bloss CS., Wineinger NE., Peters M., Boeldt DL., Ariniello L., Kim JY., Sheard J., Komatireddy R., Barrett P., Topol EJ. 2016. A prospective randomized trial examining health care utilization in individuals using multiple smartphone-enabled biosensors. PeerJ 4:e1554.

Farley T a., Dalal M a., Mostashari F., Frieden TR. 2010. Deaths Preventable in the U.S. by Improvements in Use of Clinical Preventive Services. American Journal of Preventive Medicine 38:600-609.

Fawcett T. 2015. Mining the Quantified Self: Personal Knowledge Discovery as a Challenge for Data Science. Big Data 3:249-266.

Free C., Phillips G., Watson L., Galli L., Felix L., Edwards P., Patel V., Haines A. 2013. The effectiveness of mobile-health technologies to improve health care service delivery processes: a systematic review and meta-analysis. PLoS medicine 10:e1001363.

Gibbs S. 2015. The future of wearable technology is not wearables - it's analysing the data. The Guardian.

Goss JR., Elmore JG., Lessler DS. 2003. Quality of health care delivered to adults in the United States. The New England journal of medicine 349:1866-1868; author reply 1866-1868.

Kim JY., Wineinger NE., Steinhubl SR. 2016. The Influence of Wireless Self-Monitoring Program on the Relationship Between Patient Activation and Health Behaviors, Medication Adherence, and Blood Pressure Levels in Hypertensive Patients: A Substudy of a Randomized Controlled Trial. Journal of medical Internet research 18:e116.

Krebs P., Duncan DT. 2015. Health App Use Among US Mobile Phone Owners: A National Survey. JMIR mHealth and uHealth 3:e101.

Logan AG. 2013. Transforming hypertension management using mobile health technology for telemonitoring and self-care support. The Canadian journal of cardiology 29:579-85.

National Center for Health Statistics. 2012.Underlying Cause of Death 1999-2010

Patel MS., Asch D a., Volpp KG. 2015. Wearable Devices as Facilitators, Not Drivers , of Health Behavior Change. JAMA : the journal of the American Medical Association 313:459-460.

Shaw RJ., Steinberg DM., Bonnet J., Modarai F., George A., Cunningham T., Mason M., Shahsahebi M., Grambow SC., Bennett GG., Bosworth HB. 2016. Mobile health devices: will patients actually use them? Journal of the American Medical Informatics Association.

Statista. 2016.mHealth (mobile health) industry market size projection from 2012 to 2020 (in billion U.S. dollars). Available at http://www.statista.com/statistics/295771/mhealth-globalmarket-size/ (accessed January 1, 2016).

Steinhubl SR., Wineinger NE., Patel S., Boeldt DL., Mackellar G., Porter V., Redmond JT., Muse ED., Nicholson L., Chopra D., Topol EJ. 2015. Cardiovascular and nervous system 
433 changes during meditation. Frontiers in Human Neuroscience 9:1-10.

434 Steinhubl SR., Muse ED., Topol EJ. 2015. The emerging field of mobile health. Science $435 \quad$ translational medicine 7:283rv3.

436 Swan M. 2013. The Quantified Self: Fundamental Disruption in Big Data Science and Biological 437 Discovery. Big Data 1:85-99.

438 Topol EJ. 2015. The Patient Will See You Now: The Future of Medicine is in Your Hands.

439 Virtue M a., Furne JK., Nuttall FQ., Levitt MD. 2004. Relationship between GHb Concentration 440 and Erythrocyte Survival Determined from Breath Carbon Monoxide Concentration. 441 Diabetes Care 27:931-935. 


\section{FIGURE LEGENDS}

444 Figure 1. Normalized diastolic blood pressure readings. Each box is one study individual. Points

445 are arranged along the x-axis which represents the time in days from the beginning of the study,

446 and along the y-axis which represents the normalized diastolic blood pressure reading recorded

447 at that time. The red line is the least squares regression line. Individuals are ordered left to right,

448 top to bottom according to the number of readings recorded.

449

450 Figure 2. Histogram of the lag between consecutive measures. Measures recorded near each 451 other relative to others can lead to singularity in $\boldsymbol{\Sigma}_{\mathrm{R}}$.

452

453 Figure 3. Parameter estimate and corresponding 95\% confidence interval assessing change in 454 diastolic blood pressure over the course of the study. By March 2014, three months prior to the 455 conclusion of the study, the primary study outcome (roughly $2 \mathrm{mmHg}$ decrease) was 456 observable. 
SUPPLEMENTAL LEGENDS

459 Supplemental Data 1. Diastolic blood pressure readings.

460

461 Supplemental Data 2. Systolic blood pressure readings.

462

463

Supplemental Figure 1. Histogram of the number of blood pressure readings recorded relative

464 to the time since study enrollment.

465

466

Supplemental Figure 2. Histogram of the number of blood pressure readings recorded relative 467 to the time of day (PST).

468

Supplemental Figure 3. Normalized systolic blood pressure readings. Each box is one study individual. Points are arranged along the x-axis which represents the time in days from the beginning of the study, and along the y-axis which represents the normalized diastolic blood pressure reading recorded at that time. The red line is the least squares regression line. Individuals are ordered left to right, top to bottom according to the number of readings recorded.

Supplemental Figure 4. Parameter estimate and corresponding 95\% confidence interval assessing change in systolic blood pressure over the course of the study.

Supplemental Table 1. Study participant demographics at enrollment visit $(\mathbf{n}=38)$. Values are in counts (\%) unless otherwise noted.

Supplemental Table 2. Study participant self-assessment of health $(n=38)$. Values are in 482 counts $(\%)$ unless otherwise noted. ${ }^{*}=$ values in mean (standard deviation). 


\section{Figure 1}

Normalized diastolic blood pressure readings.

Each box is one study individual. Points are arranged along the $x$-axis which represents the time in days from the beginning of the study, and along the $y$-axis which represents the normalized diastolic blood pressure reading recorded at that time. The red line is the least squares regression line. Individuals are ordered left to right, top to bottom according to the number of readings recorded. 

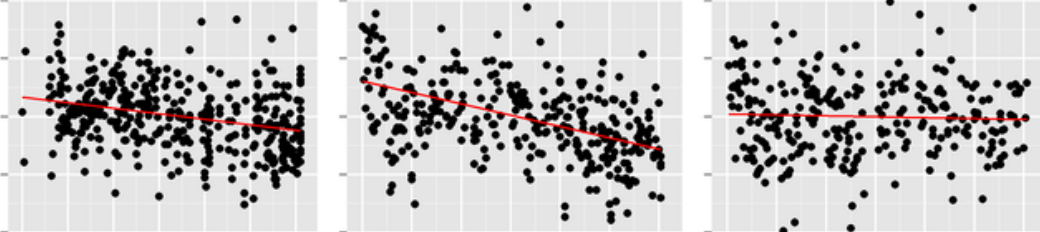

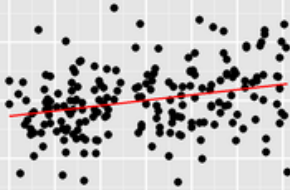
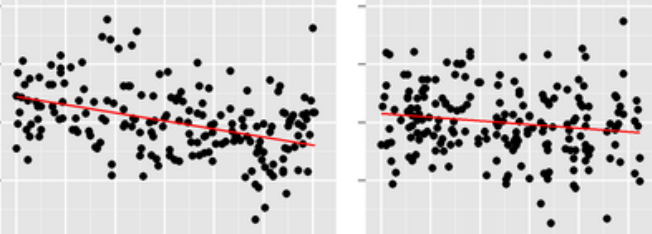

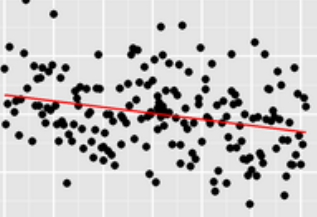
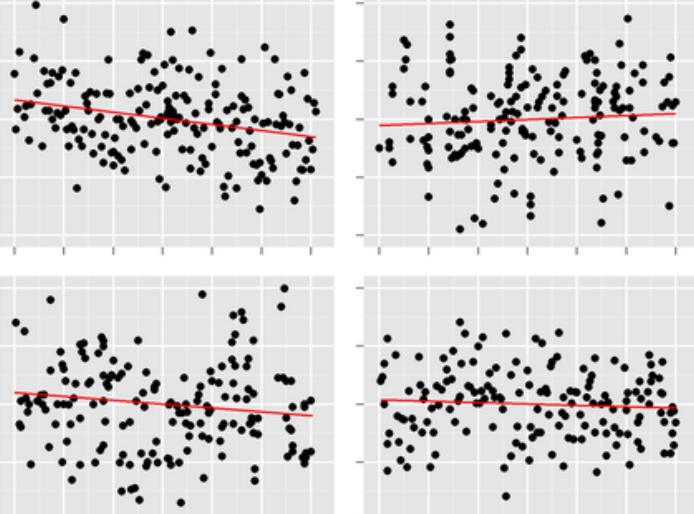

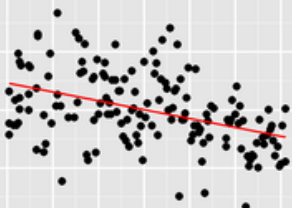
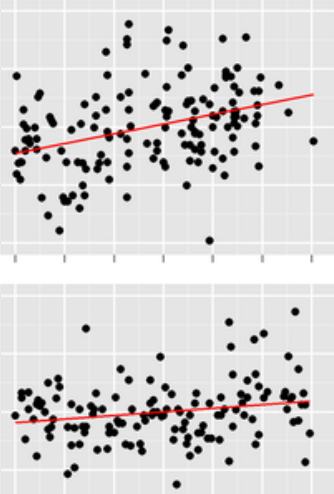
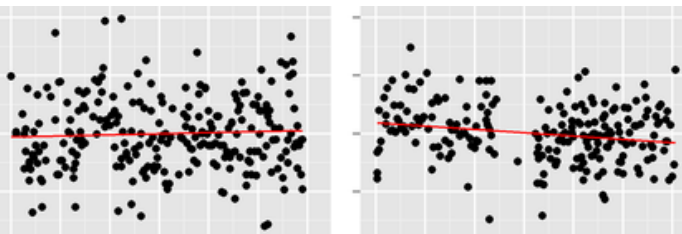

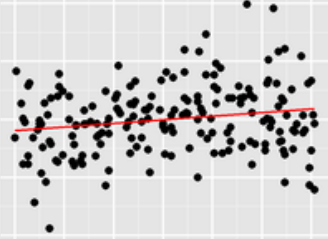

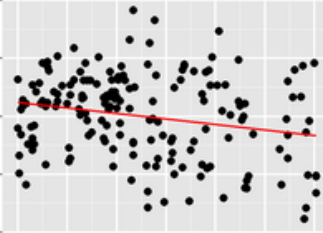

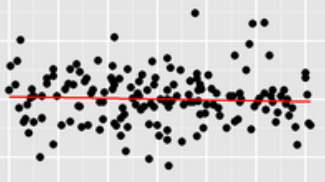
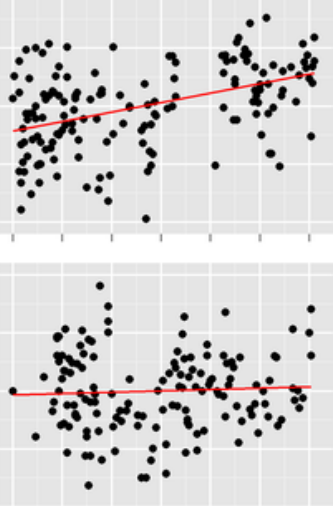

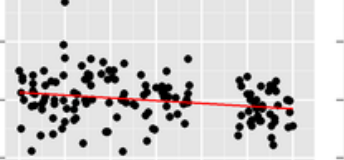

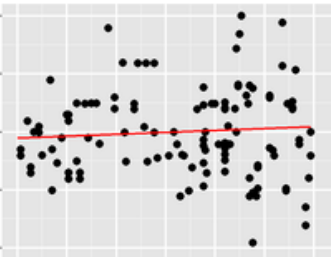

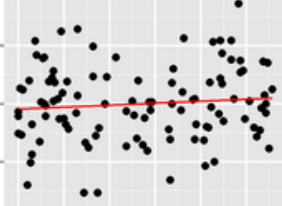

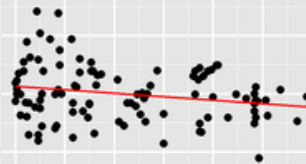

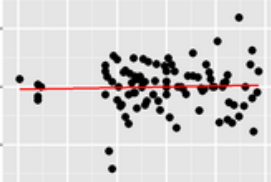

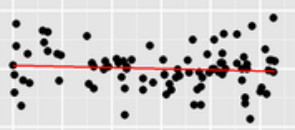

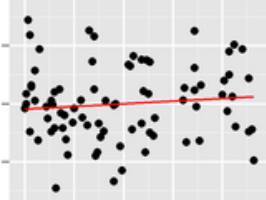

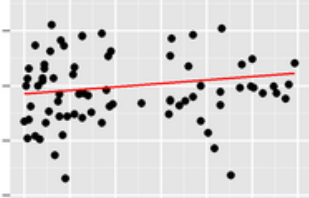

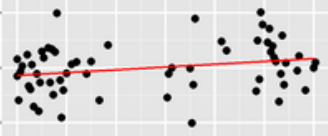


Figure 2

Histogram of the lag between consecutive measures.

Measures recorded near each other relative to others can lead to singularity in $\Sigma_{\mathrm{R}}$.

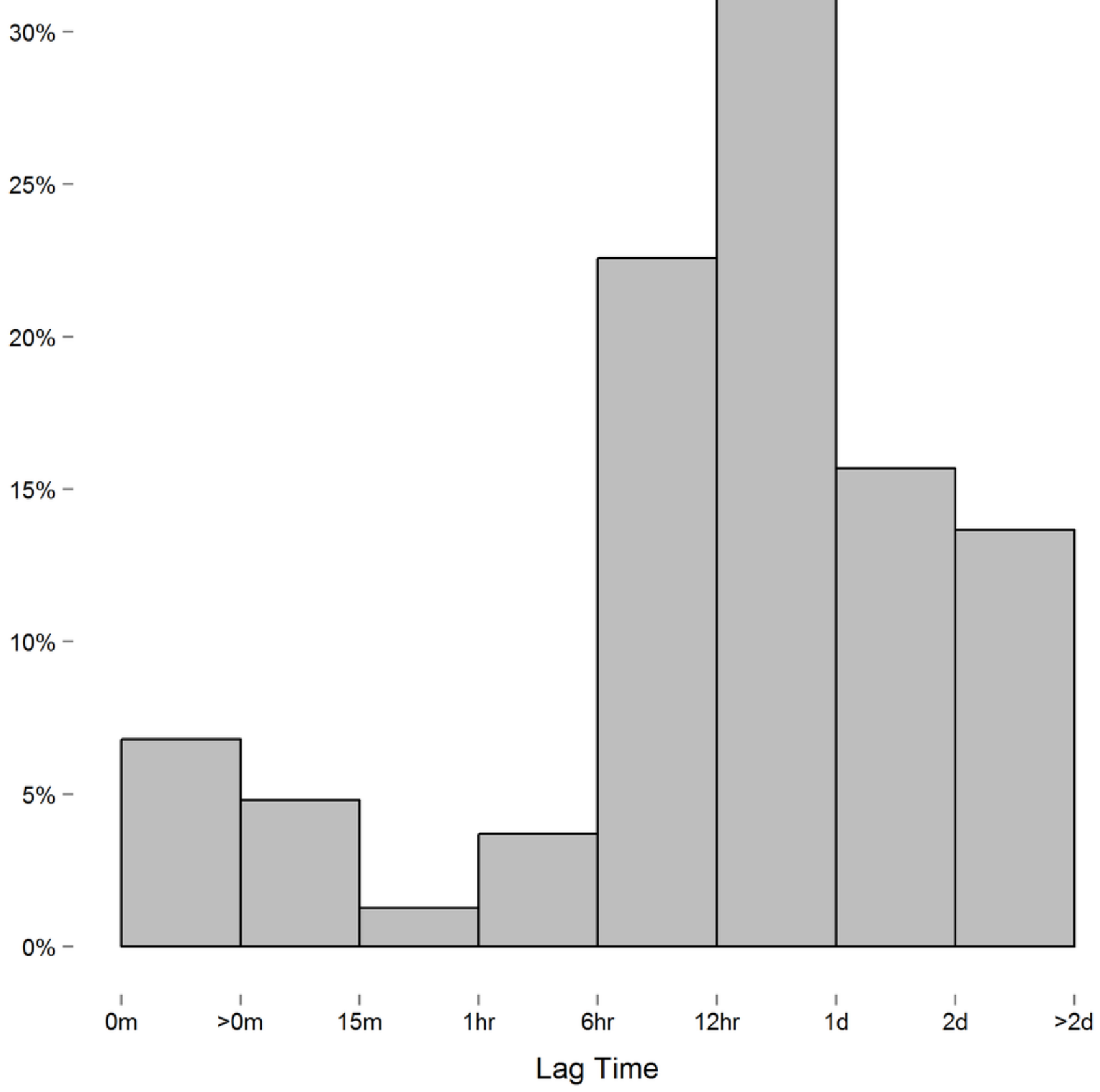




\section{Table $\mathbf{1}$ (on next page)}

Table 1. Mixed model results.

$R E=$ random effects only, $R E+A R(1)=$ first-order autoregressive model with random effects, $\mathrm{RE}+\mathrm{SP}=$ spatial power law with random effects. 
1 Table 1. Mixed model results. RE=random effects only (compound symmetric $\boldsymbol{\Sigma}_{\mathrm{R}}$ ),

$2 \mathrm{RE}+\mathrm{AR}(1)=$ first-order autoregressive model with random effects, $\mathrm{RE}+\mathrm{SP}=$ spatial power law with 3 random effects.

4

\begin{tabular}{llllll}
\hline & $\Sigma$ & AIC & BIC & Estimate $(\mathrm{Cl})$ & $\mathrm{p}$ \\
\hline Systolic & RE & 41604 & 41608 & $-2.11(-3.13,-1.09)$ & $5.19 \times 10^{-5}$ \\
& RE+AR(1) & 41495 & 41500 & $-2.11(-3.29,-0.93)$ & $4.45 \times 10^{-4}$ \\
& RE+SP & 41546 & 41550 & $-2.04(-3.11,-0.98)$ & $1.80 \times 10^{-4}$ \\
Diastolic & RE & 36725 & 36729 & $-2.04(-2.69,-1.39)$ & $9.41 \times 10^{-10}$ \\
& RE+AR(1) & 36620 & 36624 & $-2.06(-2.81,-1.31)$ & $8.17 \times 10^{-8}$ \\
& RE+SP & 36705 & 36710 & $-2.05(-2.72,-1.37)$ & $2.83 \times 10^{-9}$
\end{tabular}

5 
Figure 3

Parameter estimate and corresponding 95\% confidence interval assessing change in diastolic blood pressure over the course of the study.

By March 2014, three months prior to the conclusion of the study, the primary study outcome (roughly $2 \mathrm{mmHg}$ decrease) was observable.

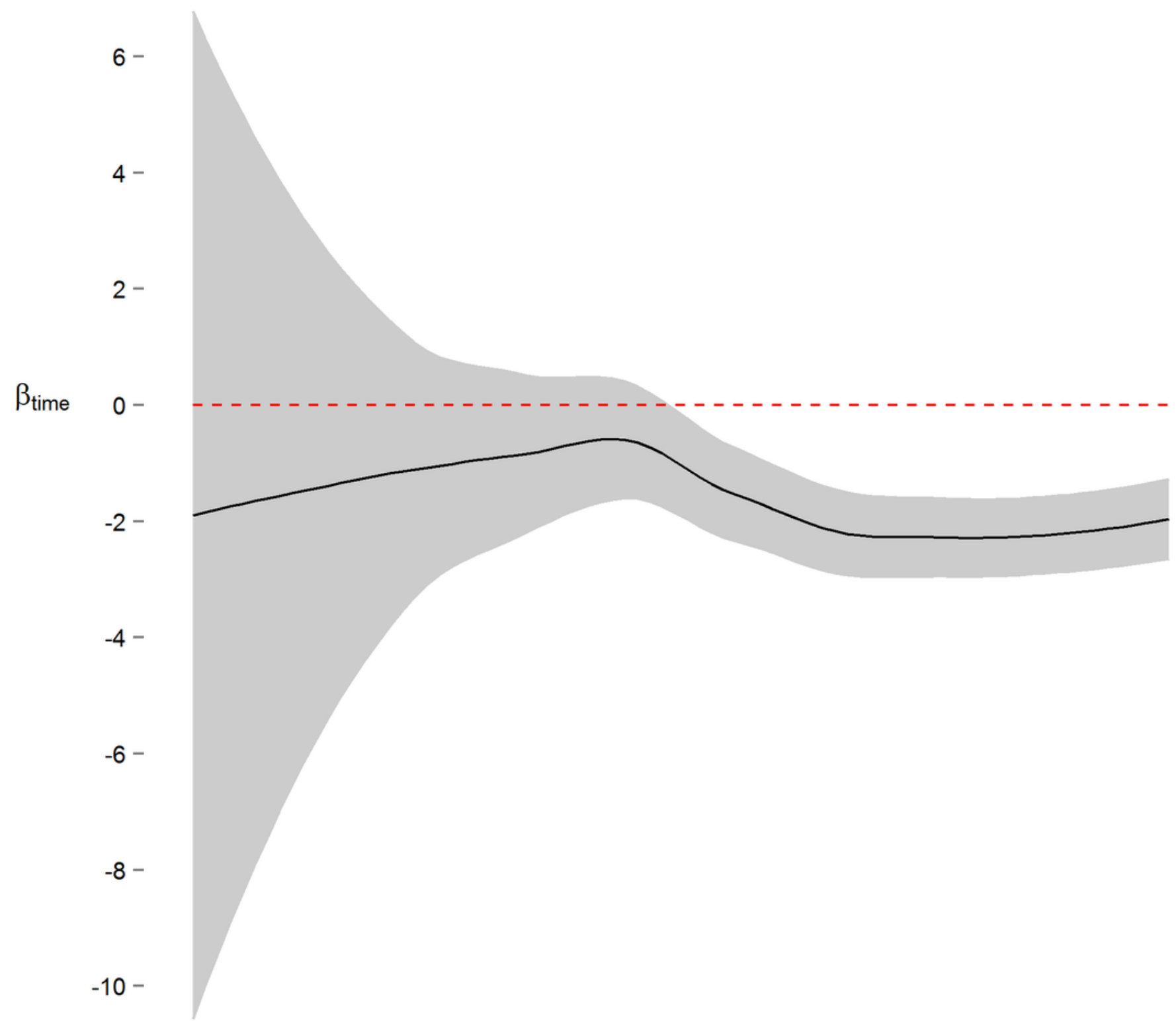

Oct 2013 